\title{
Caracterização Preliminar e Seleção de Germoplasma do Gênero Paspalum para Produção de Forragem ${ }^{1}$
}

\author{
Luiz Alberto Rocha Batista², Rodolfo Godoy²
}

\begin{abstract}
RESUMO - Com o objetivo de aumentar a disponibilidade de germoplasma para produção de forragem, 215 acessos do gênero Paspalum coletados em diferentes regiões do país foram introduzidos na Embrapa Pecuária Sudeste, situada na região central do Estado de São Paulo. Estes acessos foram avaliados e selecionados com base em suas produções, de primeiro ano, de matéria seca referentes ao desenvolvimento vegetativo durante o ano e nos períodos de alta e baixa precipitação pluvial no ano. Os acessos foram avaliados em três experimentos em delineamento experimental em blocos ao acaso, com duas repetições. A produção de matéria seca foi obtida e analisada em quatro cortes durante o ano (MSANO), os quais eram representativos do desenvolvimento dos períodos de primavera/verão (MSCHUVA) e outono/inverno (MSSECO). As análises individuais, por experimento, mostraram a presença de variabilidade entre os acessos para as características avaliadas. A análise conjunta para experimentos foi realizada pela metodologia de análise conjunta de experimentos em blocos ao acaso com tratamentos comuns (testemunhas - Brachiaria decumbens e Andropogon gayanus cv. Baetí). Em virtude de a variância obtida pela interação entre tratamentos comuns por experimentos ter sido significativa, a seleção dos acessos foi realizada dentro de cada um dos experimentos, escolhendo-se aqueles que apresentaram produção no mínimo igual à menor produção das testemunhas naquele experimento. Cinqüenta e oito acessos foram selecionados (27\%), demonstrando a possibilidade de serem obtidas cultivares com produtividade de matéria seca igual ou superior à das cultivares utilizadas como testemunhas.
\end{abstract}

Palavras-chave: acessos, ambiente, gramínea, pastagem, variabilidade genética

\section{Preliminary Characterization and Selection of Germoplasm of Genus Paspalum for Forage Production}

\begin{abstract}
With the objective to increase the germoplasm availability for forage production, 215 accessions of the genus Paspalum collected in different regions and introduced at the Embrapa Southeast - Cattle Research Center, located at the central region of the São Paulo state. These accessions were evaluated and selected based on their yields in the first year, of dry matter from the vegetative development throughout the year and during the period of high and low rain preciptation. The accesses were evaluated in three experiments using a randomized blocks desing with two replicates. Dry matter production was obtained and analyzed from four cuts during the year (DMYEAR), which were representative of the development during the spring/summer (DMRAINY) and autumn/winter (DMDRY) seasons. The individual analyses, per experiment, showed variability among the accessions for the evaluated characteristics. The analyses as a set for the experiments was analyzed by the methodology of total analyses of the experiments in a randomized blocks desing with a common treatments (controls - Brachiaria decumbens and Andropogon gayanus cv. Baetí). Due to the significant variance interaction among common treatments by experiments, the selection was done for each experiment by choosing the accesses that presented yields at least equivalent to the worst control. Fifty-eight accessions were selected (27\%), which demonstrated the possibility of obtaining cultivars with dry matter yields equal or better than the controls.
\end{abstract}

Key Words: accessions, environment, grasses, pasture, genetic variability

\section{Introdução}

A falta de alimentação adequada do gado é um dos principais fatores limitantes à produtividade pecuária no continente sul-americano. O interesse nas pesquisas sobre plantas forrageiras e a importância em selecionar novas cultivares mais produtivas têm aumentado de maneira considerável, pois a pastagem é o alimento mais abundante e barato nessas regiões.
Pesquisas têm sido implementadas para identificar gêneros, espécies e ecótipos de plantas forrageiras que estejam melhor adaptadas às condições dos diversos ecossistemas sul americanos (SCHULTZEKRAFT, 1980). No Brasil, esta atividade com gramíneas vem sendo executada na forma de coletas e intercâmbios de germoplasma exótico, principalmente do continente africano, de onde foram introduzidas espécies dos gêneros Panicum,

\footnotetext{
${ }^{1}$ Trabalho desenvolvido na Embrapa Pecuária Sudeste (CPPSE) - EMBRAPA, Caixa postal 339 - $13560-970$ - São Carlos, SP Suporte financeiro: EMBRAPA/CNPq.

2Pesquisador da EMBRAPA - CPPSE, São Carlos, SP. Bolsista do CNPq. E.mail: Ibatista@cppse.embrapa.br; godoy@cppse.embrapa.br
} 
Andropogon, Brachiaria e outras, em detrimento dos gêneros e espécies nativas da própria região, como o gênero Paspalum.

A rápida expansão das áreas cobertas por um número restrito de cultivares, especialmente quando estas se tornam contíguas e abrangem ambientes inadequados, carrega em si o perigo da vulnerabilidade genética, o que já tem trazido grandes decepções a produtores e grandes prejuízos em escala nacional. Este perigoé mais eminente, quando um único genótipo apresenta grande expansão, e isto tem acontecido com frequiência, no caso das gramíneas forrageiras apomíticas ou propagadas por via vegetativa. Os problemas causados pelo vírus do enfezamento (PSV) em pastagens de capim pangola (Digitaria decumbens) (SCHANK, 1974), os de fotossensibilização e susceptibilidade ao ataque da cigarrinha-das-pastagens em Brachiaria decumbens (PRIMO, 1975; COSENZA, 1982) e a crescente susceptibilidade de pastagens de $B$. humidicola ao ataque da cigarrinha na região Norte (DIAS FILHO, 1982) são exemplos claros da necessidade de mudança da situação atual e tornam evidente maior diversificação das opções de forrageiras, naturalmente associada à intensificação da experimentação regional, procurando explorar melhor a interação genótipo por ambiente.

A importância das espécies de Paspalum como pastagens cultivadas vem sendo evidenciada por vários autores, os quais, na sua maioria, se referem à grande variabilidade inter e intraespecífica, o que sugere ser a América do Sul o centro de origem e diversificação genética das espécies deste gênero (CHASE, 1937; BURTON, 1945; MANNETJE, 1961; BURTON, 1962; BURTON, 1967; e BATISTA e GODOY, 1997).

O trabalho teve por objetivo caracterizar e selecionar, com base na produtividade de matéria seca do primeiro ano, acessos de germoplasma do gênero Paspalum coletados em diferentes regiões do País e introduzidos na Embrapa Pecuária Sudeste.

\section{Material e Métodos}

Os trabalhos foram realizados na Embrapa Pecuária Sudeste (CPPSE), unidade da Empresa Brasileira de Pesquisa Agropecuária (EMBRAPA), localizado no município de São Carlos, região central do Estado de São Paulo a $21^{\circ} 57^{\prime}$ de Latitude Sul e $47^{\circ} 50^{\prime}$ de Longitude Oeste. Sua altitude média é de 856 metros, com precipitação pluvial anual (média de
30 anos) de $1476 \mathrm{~mm}$ de chuva e temperatura média anual de $19,8^{\circ} \mathrm{C}$. Seu ecossistema é de campos e bosques, com clima tropical de altitude. Para o desenvolvimento dos trabalhos de melhoramento genético de forrageiras do gênero Paspalum, é mantida, nesta unidade de pesquisa, uma coleção viva de acessos, principalmente do grupo botânico Plicatula, que vem sendo enriquecida por meio de coletas e intercâmbios, coordenadas e/ou executadas pela Embrapa Biotecnologia e Recursos Genéticos. Esta coleção compreende acessos oriundos de várias localidades (Tabela 1) e hoje possui 215 acessos, os quais representam, além do grupo botânico Plicatula, outros grupos deste gênero (Tabela 2).

Os acessos foram introduzidos por meio de mudas provenientes de coletas. Por desconhecer o modo reprodutivo, foi feita propagação vegetativa destas introduções - mudas ou estolões - com o objetivo de manter a integridade genética. As parcelas experimentais foram de $5 \times 2 \mathrm{~m}$, sendo a área útil de $4 \mathrm{~m}^{2}$, considerando-se as duas fileiras centrais e desprezando $0,5 \mathrm{~m}$ de suas extremidades. As mudas foram plantadas distanciadas entre si por $0,50 \mathrm{~m}$.

O primeiro experimento foi instalado em 2 de março de 1990 com 46 acessos; o segundo, em 11 de fevereiro de 1992 com 103 acessos; e o terceiro, em 25 de janeiro de 1993 com 68 acessos. Como testemunhas comuns em todos os experimentos, foram utilizados Brachiaria decumbens e Andropogon gayanus cv. Baetí. Em todos os experimentos, foi realizado um corte de uniformização trinta dias após o plantio e avaliado cada experimento por um ano agrícola.

Tabela 1 - Locais de origem dos acessos de Paspalum avaliados

Table 1 - Places of origin of the evaluated Paspalum accessions

\begin{tabular}{lcc} 
Local & $\begin{array}{c}\text { Número de acessos } \\
\text { Number }\end{array}$ & $\%$ \\
\hline Rio Grande do Sul & 91 & \\
Mato Grosso do Sul & 39 & 42,3 \\
Santa Catarina & 28 & 18,0 \\
Paraná & 25 & 13,0 \\
Mato Grosso & 07 & 11,6 \\
Goiás & 07 & 3,3 \\
Minas Gerais & 05 & 3,2 \\
Paraguai & 03 & 2,3 \\
Amapá & 03 & 1,4 \\
São Paulo & 02 & 1,4 \\
Rondônia & 02 & 1,0 \\
Tocantins & 01 & 1,0 \\
Distrito Federal & 01 & 0,5 \\
USA & 01 & 0,5 \\
Total & 215 & 0,5 \\
\hline
\end{tabular}


Rev. bras. zootec.

Tabela 2 - Grupos botânicos do gênero Paspalum representados pelos acessos avaliados Table 2 - Paspalum genus botanical groups represented by the evaluated Paspalum genus accessions

\begin{tabular}{|c|c|c|c|}
\hline $\begin{array}{l}\text { Grupo botânico } \\
\text { Botanical groups }\end{array}$ & $\begin{array}{l}\text { Espécie } \\
\text { Specie }\end{array}$ & $\begin{array}{l}\text { Número de acessos } \\
\text { Number of accessions }\end{array}$ & $\begin{array}{l}\text { Percentual da espécie } \\
\text { Percentage of species }\end{array}$ \\
\hline \multirow[t]{8}{*}{ Plicatula } & P.plicatulum & 22 & 10,2 \\
\hline & P. compressifolium & 14 & 6,5 \\
\hline & $P$. yaguaronense & 11 & 5,1 \\
\hline & P. guenoarum & 5 & 2,3 \\
\hline & P. oteroi & 3 & 1,4 \\
\hline & P. nicorae & 2 & 0,9 \\
\hline & P. atratum & 1 & 0,5 \\
\hline & Paspalum sp. & 112 & 52,0 \\
\hline \multirow[t]{4}{*}{ Notata } & $P$. notatum & 10 & 4,6 \\
\hline & P. ionanthum & 2 & 0,9 \\
\hline & P. subciliatum & 1 & 0,5 \\
\hline & P. cromyorrhizon & 2 & 0,9 \\
\hline \multirow[t]{3}{*}{ Caespitosa } & $P$. indecorum & 3 & 1,4 \\
\hline & P. maritimum & 3 & 1,5 \\
\hline & P. chacoense & 2 & 0,9 \\
\hline \multirow[t]{2}{*}{ Malacophylla } & P. simplex & 2 & 0,9 \\
\hline & P. malacophyllum & 1 & 0,5 \\
\hline Modesta & P. modestum & 5 & 2,3 \\
\hline Livida & P. lividum & 3 & 1,4 \\
\hline \multirow[t]{3}{*}{ Virgata } & P. conspersum & 3 & 1,4 \\
\hline & P.regnellii & 1 & 0,5 \\
\hline & Paspalum sp. & 1 & 0,5 \\
\hline Fasciculata & P.fasciculatum & 2 & 0,9 \\
\hline Quadrifaria & P. coryphaeum & 1 & 0,5 \\
\hline Conjugata & P. conjugatum & 1 & 0,5 \\
\hline Disticha & P. vaginatum & 1 & 0,5 \\
\hline Ovalia & P. ovale & 1 & 0,5 \\
\hline Total & & 215 & 100 \\
\hline
\end{tabular}

A característica avaliada foi a produção de matéria seca do primeiro ano, obtida por meio de quatro cortes referentes ao desenvolvimento de inverno, primavera, verão e outono. A produção do período seco (MSSECO) foi representada pela soma das produções obtidas nos cortes realizados no outono e inverno, que refletem o desempenho do germoplasma durante o período de precipitação pluvial baixa; a produção do período chuvoso (MSCHUVA), pela soma das produções obtidas nos cortes realizados na primavera e verão, que reflete o desempenho do germoplasma durante o período de precipitação pluvial elevada; e a produção total por ano (MSANO), obtida pela soma dos quatro cortes. Os valores obtidos foram transformados em quilogramas de matéria seca por hectare (kg MS/ha).

O experimento de campo foi instalado em Latossolo Vermelho Amarelo (LVA) álico, cujas características físicas e químicas estão apresentadas na Tabela 3.

O delineamento experimental foi o de blocos ao acaso com duas repetições, sendo que a análise da variância nas características avaliadas, por experimento, foi realizada de acordo com o seguinte modelo linear fixo: $y_{i j k}=\mu+R_{i}+A_{j}+e_{i j}$, em que $\mu$ é efeito da média geral; $\mathrm{R}_{\mathrm{i}}$, efeito da repetição $\mathrm{i} ; \mathrm{A}_{\mathrm{j}}$, efeito fixo do acesso $\mathrm{j}$; e $\mathrm{e}_{\mathrm{ij}}$, efeito do erro experimental do acesso $\mathrm{j}$ na repetição $\mathrm{i}$.

A análise conjunta foi realizada pela metodologia de análises conjunta de experimentos em blocos ao acaso com tratamentos comuns (testemunhas) GOMES (1978). Esse tipo de análise permite o cálculo do coeficiente de ajuste das médias por tratamento dentro de cada experimento em relação aos tratamentos comuns. Foi usado o teste Tukey a $5 \%$ para os contrastes entre as médias dos acessos. As médias para produção dos períodos seco e chuvoso foram transformadas em porcentagem em relação à produção total do ano. A seleção dos acessos mais produtivos foi feita com base na produção obtida pela testemunha menos produtiva.

\section{Resultados e Discussão}

Os resultados das variabilidades fenotípicas obtidas nos experimentos 1, 2 e 3 estão apresentados na Tabela 4. A fonte de variação acessos apresentou 
BATISTA e GODOY

Tabela 3 - Características do solo onde foram realizados os experimentos Table 3 - Characteristics of the soil where the experiments were conducted

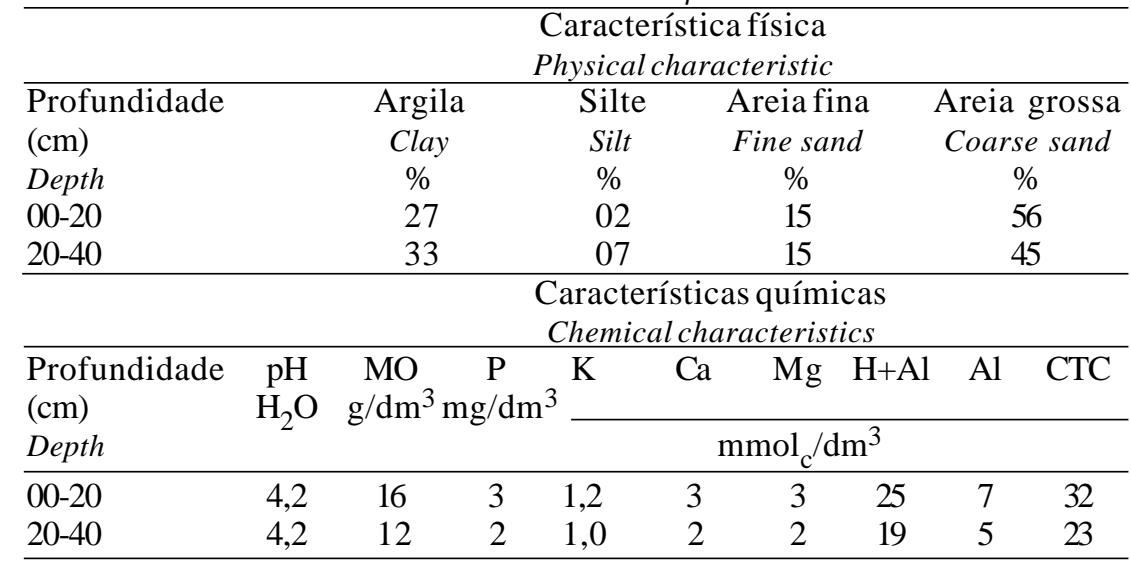

Tabela 4 - Análise da variância ( $F V=$ fonte de variação, $G L=$ graus de liberdade, $Q M=$ quadrado médio e $\mathrm{F}=$ teste de Snedecor) por experimento (E1, E2 e E3), para as características MSANO, MSSECO eMSCHUVA

Table 4 - Analysis of variance $(S V=$ source of variation, $D F=$ degrees of freedom, $M S=$ mean square and $F=S$ nedecor test) per experiment (E1, E2 and E3) for the DMYEAR, DMDRY and DMRAINY characteristics

\begin{tabular}{|c|c|c|c|c|c|c|c|c|c|}
\hline \multirow[t]{2}{*}{$\begin{array}{l}\overline{\mathrm{FV}} \\
S V\end{array}$} & \multicolumn{3}{|c|}{$\begin{array}{l}\text { GL } \\
D F\end{array}$} & \multicolumn{3}{|c|}{$\begin{array}{l}\text { QM } \\
M S\end{array}$} & \multicolumn{3}{|c|}{$\mathrm{F}$} \\
\hline & E1 & E2 & E3 & E1 & $\mathrm{E} 2$ & $\mathrm{E3}$ & E1 & $\mathrm{E} 2$ & E3 \\
\hline & & & & & $\begin{array}{l}\text { SANO } \\
\text { IYEAR }\end{array}$ & & & & \\
\hline $\begin{array}{l}\text { Blocos } \\
\text { Blocks }\end{array}$ & 1 & 1 & 1 & 3,57 & 19,53 & 18,21 & 0,84 & $4,08 *$ & 1,19 \\
\hline $\begin{array}{l}\text { Acessos } \\
\text { Accessions }\end{array}$ & 47 & 104 & 69 & 58,22 & 67,27 & 162,18 & $13,75^{* *}$ & $14,06 * *$ & $10,54 * *$ \\
\hline $\begin{array}{l}\text { Resíduo } \\
\text { Error }\end{array}$ & 47 & 104 & 69 & 4,23 & 4,79 & 15,34 & & & \\
\hline $\begin{array}{l}\text { Média (t/ha) } \\
\text { Mean }\end{array}$ & 11,11 & 8,73 & 12,56 & & & & & & \\
\hline $\mathrm{CV} \%$ & 18,52 & 25,07 & 31,21 & & $\begin{array}{l}\text { SSECO } \\
M D R Y\end{array}$ & & & & \\
\hline $\begin{array}{l}\text { Blocos } \\
\text { Blocks }\end{array}$ & 1 & 1 & 1 & 0,58 & 0,05 & 2,91 & 1,17 & 0,07 & 1,33 \\
\hline $\begin{array}{l}\text { Acessos } \\
\text { Accessions }\end{array}$ & 47 & 104 & 69 & 5,04 & 9,62 & 22,08 & $10,23 * *$ & $13,77 * *$ & $10,08 * *$ \\
\hline $\begin{array}{l}\text { Resíduo } \\
\text { Error }\end{array}$ & 47 & 104 & 69 & 0,49 & 0,70 & 2,19 & & & \\
\hline $\begin{array}{l}\text { Média (t/ha) } \\
\text { Mean }\end{array}$ & 3,68 & 2,65 & 4,76 & & & & & & \\
\hline $\mathrm{CV} \%$ & 19,09 & 31,51 & 31,09 & $\begin{array}{c}\mathrm{MS} \\
D I\end{array}$ & $\begin{array}{l}\text { CHUVA } \\
\text { RAINY }\end{array}$ & & & & \\
\hline $\begin{array}{l}\text { Blocos } \\
\text { Blocks }\end{array}$ & 01 & 1 & 1 & 1,28 & 21,57 & 6,56 & 0,50 & $7,88 * *$ & 0,77 \\
\hline $\begin{array}{l}\text { Acessos } \\
\text { Accessions }\end{array}$ & 47 & 104 & 69 & 33,17 & 29,53 & 72,59 & $12,88 * *$ & $10,79 * *$ & $8,56 * *$ \\
\hline $\begin{array}{l}\text { Resíduo } \\
\text { Error }\end{array}$ & 47 & 104 & 69 & 2,58 & 2,73 & 8,48 & & & \\
\hline $\begin{array}{l}\text { Média }(t / h a) \\
\text { Mean }\end{array}$ & 7,43 & 6,08 & 7,79 & & & & & & \\
\hline CV\% & 21,59 & 27,23 & 37,36 & & & & & & \\
\hline
\end{tabular}

*, ** Significativo a 5 e $1 \%$ de probabilidade, respectivamente, pelo teste $\mathrm{F}$.

${ }^{*},{ }^{* *}$ Significant at 5 and $1 \%$ of probability, respectively, by $F$ test. 
valores de F significativos a $1 \%$ de probabilidade, nos três experimentos, para as características de produção de matéria seca no ano, no período de baixa e alta precipitação, indicando a presença de variabilidade significativa. O efeito de repetição foi significativo somente no experimento 2 para as características de produção total por ano $(\mathrm{P}<0,05)$ e produção no período de máxima precipitação $(\mathrm{P}<0,01)$, mostrando que somente duas repetições não foram suficientes para reduzir o efeito ambiental de local, quando se tem número grande de acessos (105) a ser avaliado. As médias de produção total foram 11109, 8727 e 12555 $\mathrm{kg} / \mathrm{ha}$ nos experimentos 1,2 e 3, respectivamente. Deste total, 33, 30 e $37 \%$ representam a produção relativa ao período de baixa precipitação e seu complemento 67,70 e $63 \%$, devido à produção no período de alta precipitação, respectivamente, para os experimentos 1, 2 e 3 . Estes resultados reforçam a comprovação de que o total da produção anual de matéria seca em várias gramíneas tropicais é composto de 30 a $35 \%$, devido ao desenvolvimento no período de baixa precipitação e, de 65 a 70\%, devido ao desenvolvimento no período de alta precipitação (Carnevalli e Silva, 1998; citados por RODRIGUES et al. 1998). Esta mesma tendência foi obtida nas testemunhas Andropogon gayanus e Brachiaria decumbens apresentadas na Tabela 6. Deve-se ressaltar que estas relações de biomassa foram obtidas em avaliações de primeiro ano de implantação das pastagens.

Os resultados da análise conjunta para as características de MSANO, MSSECO e MSCHUVA estão apresentados na Tabela 5, os quais mostram significância $(\mathrm{P}<0,01 \%)$ para os efeitos de experimento e acessos (ajustados por tratamentos comunstestemunhas) e interação testemunhas por experimentos (Test. x Exp.). A significância da interação testemunha por experimento indicou a necessidade em interpretar os resultados dentro de cada experimento, pois os efeitos ambientais entre estes foram elevados, impossibilitando a interpretação de forma única para todos acessos avaliados. Portanto, a seleção dos acessos foi feita dentro de cada um dos experimentos. Dessa forma, para maior eficiência seletiva, os acessos selecionados devem ser avaliados em conjunto em vários ambientes (locais e anos), visando a seleção para a estabilidade produtiva, em função da variação ambiental.

A Tabela 6 apresenta as produtividades de matéria seca do primeiro ano e as proporções relativas à produção total do ano nos períodos chuvoso e seco para os acessos selecionados e as testemunhas Andropogon gayanus e Brachiaria decumbens por experimento. No experimento 1, a maior produtividade anual de matéria seca foi obtida pelo acesso BRA-003913, com $23.492 \mathrm{~kg} / \mathrm{ha}, 32,6 \%$ acima da menor testemunha Brachiaria decumbens. Neste experimento, foram selecionados 15 acessos, 33\% dos acessos avaliados. Destes, quatro acessos foram selecionados, por apresentarem produções de matéria seca elevada nos três períodos considerados: BRA-003824 - P. guenoarum; BRA-003913Paspalum sp; BRA-009661 Paspalum sp; BRA009695 Paspalum sp. No experimento 2, a maior produtividade foi obtida pelo acesso BRA-009610 $P$. atratum, com $22.142 \mathrm{~kg}$ MS/ha, 2,3\% acima da menor testemunha Andropogon gayanus. Neste experimento, foram selecionados 15 acessos, $15 \%$ dos acessos avaliados. Destes, oito acessos foram selecionados, por apresentarem elevada produção anual e no período chuvoso. Nenhum acesso foi selecionado, por apresentar produção no período seco acima da testemunha de menor produção. No

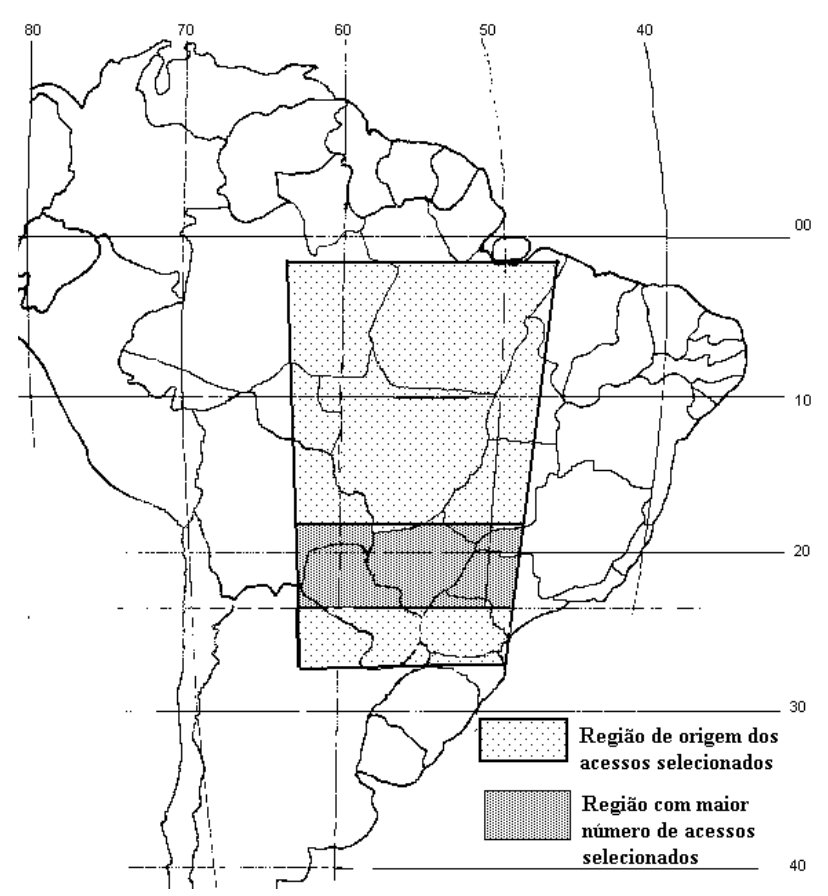

Figura 1 - Quadrante obtido pelas coordenadas de latitude e longitude limites dos locais em que foram coletados os acessos de Paspalum selecionados para produção de matéria seca.

Figure 1 - Quadrant obtained by the latitude and longitude limits coordinates from the sites, in which the accessions of Paspalum selected by dry matterproduction were collected. 
experimento 3, a maior produtividade foi obtida pelo acesso BRA-011401, com $37.359 \mathrm{~kg} \mathrm{~ms} / \mathrm{ha}, 21 \%$ acima da testemunha de menor produção Brachiaria decumbens. Neste experimento, foram selecionados 28 acessos, $41 \%$ dos acessos avaliados. Destes, três acessos foram selecionados, por apresentarem produções superiores nos três períodos avaliados: BRA010383 Paspalum sp, BRA-011401 P. yaguaronense e BRA-012700 Paspalum sp.

Do total de 215 acessos, 58 foram selecionados com base na produção de matéria seca do primeiro ano, os quais estão apresentados na Tabela 7, demonstrando a possibilidade de se obterem cultivares com produtividade de matéria seca igual ou superior às cultivares de Brachiaria decumbens ou de Andropogon gayanus cv. Baetí, principais forrageiras das regiões Centro Oeste e Sudeste.

As diferenças de produção de matéria seca (Tabela 6) observadas entre acessos de uma mesma espécie (Tabela 7), mesmo dentro daqueles selecionados como P. guenoarum acessos: BRA-003824; BRA066572 e BRA-014851, indicam a existência de variabilidade intraespecífica significativas para a característica produção de matéria seca. Este tipo de variabilidade em espécies do gênero Paspalum foi demonstrada por TISCHLER e BURSON (1995) e BATISTA e GODOY (1997) em $P$. notatum e STRAPASON (1997) em $P$. plicatulum e $P$. guenoarum. A variabilidade estudada por esses autores refletem diferenças observadas em características morfológicas. TISCHLER e BURSON (1995) sugerem que estas variações sejam atribuídas a diferenças que ocorrem com o nível de ploidia entre os acessos. No caso deste estudo, as variações observadas especificamente entre acessos de $P$. guenoarum não são devidas a variações no nível de ploidia, pois estes acessos apresentam o mesmo nível com $2 n=40$ (TAKAYAMA et al., 1998), o que sugere ser uma variação de base genética.

Todos os acessos selecionados no presente trabalho são provenientes do quadrante apresentado no mapa da Figura 1. Dentro deste polígono, observa-se que a área em destaque, formada pelas latitudes $19^{\circ} \mathrm{S}$ a $22^{\circ} \mathrm{S}$ e longitudes $47^{\circ} \mathrm{W}$ a $63^{\circ} \mathrm{W}$, contém 24 acessos selecionados. Este resultado dá indicação de que futuras expedições de coleta de acessos do gênero Paspalum para o sudeste brasileiro deverão considerar esta região como prioritária.

Entre os acessos selecionados (Tabela 7), encontra-se o acesso BRA-009610 de $P$. atratum, denominado de variedade "Suerte" por KALMBACHER et al. (1997), o qual, quando utilizado em pastejo rotacionado com diferimento de 20 a 40 dias, promove elevadas produções de matéria seca de adequados valores nutritivos. Sua produtividade anual de matéria seca foi de $14,8 \mathrm{t} / \mathrm{ha}$, concentrada principalmente na época das chuvas.

As análises de correlação apresentaram relação linear positiva e significativa $(\mathrm{P}<0,01)$ entre a

Tabela 5 - Análise conjunta da variância $(F V=$ fonte de variação, $G L=$ graus de liberdade, $Q M=$ quadrado médio e $F=$ teste de Snedecor), para as características MSANO, MSSECO e MSCHUVA

Table 5 - Combined analysis of variance $(S V=$ source of variation, $D F=$ degrees of freedom, $M S=$ mean square and $F=S n e d e c o r$ test) for the DMYEAR, DMDRY and DMRAINY characteristics

\begin{tabular}{|c|c|c|c|c|c|c|c|}
\hline \multirow{4}{*}{$\begin{array}{l}\mathrm{FV} \\
S V\end{array}$} & \multirow{4}{*}{$\begin{array}{l}\text { GL } \\
D F\end{array}$} & \multirow{2}{*}{\multicolumn{3}{|c|}{$\begin{array}{l}\mathrm{QM} \\
M S\end{array}$}} & \multirow{2}{*}{\multicolumn{3}{|c|}{$\mathrm{F}$}} \\
\hline & & & & & & & \\
\hline & & MSANO & MSSECO & $\overline{\text { MSCHUVA }}$ & MSANO & MSSECO & MSCHUVA \\
\hline & & DMYEAR & $D M D R Y$ & DMRAINY & DMYEAR & $D M D R Y$ & DMRAINY \\
\hline$\overline{\text { Experimento(Exp) }}$ & 2 & 642,36 & 187,74 & 141,09 & $86,16^{* *}$ & $167,27 * *$ & $35,52 * *$ \\
\hline Experiment $(\operatorname{Exp})$ & & & & & & & \\
\hline Blocos (Exp) & 3 & 13,77 & 1,18 & 9,80 & 1,85 & 1,05 & 2,49 \\
\hline Blocks (Exp) & & & & & & & \\
\hline Acessos/ajustados & 218 & 95,98 & 12,67 & 44,21 & $12,87 * *$ & $11,29 * *$ & $11,25 * *$ \\
\hline Adjusted acessions & & & & & & & \\
\hline Testemunha $x$ Experimento & 2 & 57,81 & 3,80 & 63,53 & $7,75 * *$ & $3,39 * *$ & $16,17 * *$ \\
\hline Control $x$ Experiment & & & & & & & \\
\hline Resíduo & 220 & 7,46 & 1,12 & 3,93 & & & \\
\hline Pooled error & & & & & & & \\
\hline Total & 445 & & & & & & \\
\hline Média (kg MS/ha) & 10.442 & 3535 & 6907 & & & & \\
\hline Mean $(\mathrm{kg} D M / h a)$ & & & & & & & \\
\hline $\mathrm{CV} \%$ & 26,16 & 29,95 & 28,70 & & & & \\
\hline
\end{tabular}

** Significativo a $1 \%$ de probabilidade pelo teste $\mathrm{F}$.

${ }^{*}$ Significant at $1 \%$ of probability by $F$ test. 
Rev. bras. zootec.

Tabela 6 - Produção de matéria seca no ano (MSANO) e percentual de produção, nos períodos chuvoso (MSCHUVA) e seco (MSSECO) dos acessos mais produtivo de Paspalum, nas avaliações de primeiro ano para cada experimento realizado em São Carlos, SP

Table 6 - Dry matter yield in the year (DMYEAR) and percentage of production in the rainy period (DMRAINY) and dry period (DMDRY) of the most productive accession of Paspalum, in the first year evaluation, for each experiment carried out in São Carlos, SP

\begin{tabular}{|c|c|c|c|}
\hline Acesso & $\operatorname{MSANO}(\mathrm{kg} / \mathrm{ha})^{1}$ & $\operatorname{MSCHUVA}(\%)$ & $\operatorname{MSSECO}(\%)$ \\
\hline Accession & DMYEAR & DMRAINY & $D M D R Y$ \\
\hline \multicolumn{4}{|c|}{ Primeiro experimento } \\
\hline \multicolumn{4}{|c|}{ First experiment } \\
\hline A. gayanus ${ }^{2}$ & $25.281^{\mathrm{a}}$ & 77,79 & 22,21 \\
\hline BRA-003913 & $23.492^{\mathrm{a}}$ & 72,96 & 27,04 \\
\hline BRA-003824 & $21.842^{\mathrm{a}}$ & 69,42 & 30,58 \\
\hline BRA-009695 & $18.510^{\mathrm{ab}}$ & 62,09 & 37,91 \\
\hline BRA-008311A & $18.327^{\mathrm{b}}$ & 73,51 & 26,49 \\
\hline BRA-009661 & $17.733^{b}$ & 65,26 & 34,74 \\
\hline B. decumbens ${ }^{2}$ & $17.717^{\mathrm{b}}$ & 66,67 & 33,33 \\
\hline BRA-009687 & $16.452^{\mathrm{b}}$ & 73,23 & 26,77 \\
\hline BRA-010537 & $15.786^{\mathrm{b}}$ & 70,29 & 29,71 \\
\hline BRA-009652 & $15.740^{\mathrm{b}}$ & 71,96 & 28,04 \\
\hline BRA-003841 & $13.807^{\mathrm{b}}$ & 69,59 & 30,41 \\
\hline BRA-009415 & $13.807^{\mathrm{b}}$ & 65,23 & 34,77 \\
\hline BRA-010596 & $13.719^{\mathrm{b}}$ & 72,43 & 27,57 \\
\hline BRA-009229 & $13.504^{\mathrm{b}}$ & 57,93 & 42,07 \\
\hline BRA-003638 & $13.233^{\mathrm{b}}$ & 76,93 & 23,07 \\
\hline BRA-007498 & $12.875^{\mathrm{b}}$ & 58,79 & 41,21 \\
\hline BRA-001503 & $11.955^{\mathrm{b}}$ & 54,69 & 45,31 \\
\hline \multicolumn{4}{|c|}{ Segundo experimento } \\
\hline \multicolumn{4}{|c|}{ Second experiment } \\
\hline B. decumbens ${ }^{2}$ & $28.303^{\mathrm{a}}$ & 63,30 & 36,70 \\
\hline BRA-009610 & $22.142^{\mathrm{ab}}$ & 74,43 & 25,57 \\
\hline A. gayanus ${ }^{2}$ & $21.643^{\mathrm{abc}}$ & 59,49 & 40,51 \\
\hline BRA-000841 & $21.466^{\mathrm{bc}}$ & 63,51 & 36,49 \\
\hline BRA-007480B & $21.317^{\mathrm{bc}}$ & 72,03 & 27,97 \\
\hline BRA-010511 & $19.544^{\mathrm{bc}}$ & 74,32 & 25,68 \\
\hline BRA-014851 & $19.488^{\mathrm{bc}}$ & 70,49 & 29,51 \\
\hline BRA-010464 & $18.020^{\mathrm{c}}$ & 71,98 & 28,02 \\
\hline BRA-009032 & $17.159^{\mathrm{c}}$ & 59,86 & 40,14 \\
\hline BRA-012424 & $17.148^{c}$ & 64,33 & 35,67 \\
\hline BRA-006572 & $15.669^{c}$ & 69,30 & 30,70 \\
\hline BRA-010260 & $15.296^{c}$ & 78,01 & 21,99 \\
\hline BRA-014770 & $15.276^{\mathrm{c}}$ & 66,48 & 33,52 \\
\hline BRA-012416 & $14.791^{\mathrm{c}}$ & 72,73 & 27,27 \\
\hline BRA-008923 & $14.751^{\mathrm{c}}$ & 72,25 & 27,75 \\
\hline BRA-008508B & $14.164^{c}$ & 75,13 & 24,87 \\
\hline BRA-015067 & $12.370^{\mathrm{c}}$ & 84,07 & 15,94 \\
\hline \multicolumn{4}{|c|}{ Terceiro experimento } \\
\hline \multicolumn{4}{|c|}{ Third experiment } \\
\hline BRA-011401 & $37.359 \mathrm{a}$ & 68,43 & 31,57 \\
\hline A. gayanus ${ }^{2}$ & $26.075 \mathrm{ab}$ & 69,51 & 30,49 \\
\hline BRA-010383 & $22.179 b$ & 58,57 & 41,43 \\
\hline BRA-019186 & $22.116 b$ & 72,01 & 27,99 \\
\hline BRA-012700 & $21.364 b c$ & 65,41 & 34,59 \\
\hline BRA-009679 & $21.132 b c$ & 74,63 & 25,37 \\
\hline B. decumbens ${ }^{2}$ & $20.968 b c$ & 53,79 & 46,21 \\
\hline BRA-009181 & $20.837 b c$ & 61,84 & 38,16 \\
\hline BRA-011517 & $20.732 b c$ & 60,42 & 39,58 \\
\hline BRA-012645 & $20.621 b c$ & 56,20 & 43,80 \\
\hline BRA-012718 & $20.116 b c$ & 58,28 & 41,72 \\
\hline BRA-012866 & $19.357 \mathrm{bc}$ & 60,93 & 39,07 \\
\hline BRA-011681 & $18.568 \mathrm{bc}$ & 57,08 & 42,92 \\
\hline BRA-005541 & $18.545 \mathrm{bc}$ & 61,94 & 38,06 \\
\hline BRA-009407 & $18.467 b c$ & 51,35 & 48,65 \\
\hline BRA-008630 & $18.136 b c$ & 53,96 & 46,04 \\
\hline
\end{tabular}


BATISTA e GODOY

Continuação da Tabela 6...

\begin{tabular}{lccc}
\hline $\begin{array}{l}\text { Acesso } \\
\text { Accession }\end{array}$ & $\begin{array}{c}\text { MSANO }(\mathrm{kg} / \mathrm{ha})^{1} \\
\text { DMYEAR }\end{array}$ & $\begin{array}{c}\text { MSCHUVA(\%) } \\
\text { DMRAINY }\end{array}$ & $\begin{array}{c}\text { MSSECO }(\%) \\
\text { DMDRY }\end{array}$ \\
\hline BRA-008486 & $18.062 \mathrm{bc}$ & 56,93 & 43,07 \\
BRA-009628 & $17.761 \mathrm{bc}$ & 62,07 & 37,93 \\
BRA-012483 & $17.465 \mathrm{bc}$ & 62,22 & 37,78 \\
BRA-009644 & $16.685 \mathrm{bc}$ & 62,56 & 37,44 \\
BRA-012874 & $16.115 \mathrm{bc}$ & 67,12 & 32,88 \\
BRA-008613 & $15.732 \mathrm{c}$ & 67,55 & 32,45 \\
BRA-009113 & $15.503 \mathrm{c}$ & 57,37 & 42,63 \\
BRA-014630 & $15.116 \mathrm{c}$ & 57,02 & 42,98 \\
BRA-012556 & $14.586 \mathrm{c}$ & 74,46 & 25,54 \\
BRA-012742 & $13.986 \mathrm{c}$ & 67,56 & 32,44 \\
BRA-013871 & $13.480 \mathrm{c}$ & 47,94 & 52,06 \\
BRA-008851 & $12.737 \mathrm{c}$ & 44,80 & 55,20 \\
BRA-013951 & $12.552 \mathrm{c}$ & 40,91 & 59,09 \\
BRA-011487 & $11.594 \mathrm{c}$ & 43,01 & 56,99 \\
\hline
\end{tabular}

${ }^{1}$ Médias seguidas de letra diferentes são diferentes $(P<0,05)$ pelo teste Tukey.

Means followed by different letters are different $(P<.05)$ by Tukey test.

Tabela 7 - Espécies e grupo botânico dos acessos selecionados com base na sua produção de matéria seca do primeiro ano de avaliação

Table 7 - Species and botanical group of the accessions selected based on dry matter yield in the first year of the evaluation

\begin{tabular}{|c|c|c|c|}
\hline $\begin{array}{l}\text { Acesso } \\
\text { Accession }\end{array}$ & $\begin{array}{l}\text { Espécie } \\
\text { Specie }\end{array}$ & $\begin{array}{l}\text { Grupo botânico } \\
\text { Botanical group }\end{array}$ & $\begin{array}{c}\text { Local da coleta } \\
\text { Collection site }\end{array}$ \\
\hline BRA-011401 & P. yaguaronense & Plicatula & Lagoa Vermelha, $\mathrm{F}$ \\
\hline BRA-003913 & Paspalum sp & Plicatula & Miranda, MS \\
\hline BRA-010383 & Paspalum sp & Plicatula & Corumbá, MS \\
\hline BRA-009610 & P. atratum & Quadrifaria & Terenos, MS \\
\hline BRA-019186 & P. regnellii & Quadrifaria & Rio Claro, SP \\
\hline BRA-003824 & P. guenoarum & Plicatula & Bela Vista, MS \\
\hline BRA-000841 & P. coryphaeum & Quadrifaria & Anaurilândia, MS \\
\hline BRA-012700 & Paspalum sp & Virgata & Dourados, MS \\
\hline BRA-007480B & P. conspersum & Virgata & Corumbá, MS \\
\hline BRA-009679 & Paspalum sp & Plicatula & Aquidauana, MS \\
\hline BRA-009181 & P.plicatulum & Plicatula & São Gabriel, RS \\
\hline BRA-011517 & Paspalum $s p$ & Plicatula & Bom Jesus, RS \\
\hline BRA-012645 & Paspalum sp & Plicatula & Dourados, MS \\
\hline BRA-012718 & Paspalum sp & Plicatula & Dourados, MS \\
\hline BRA-010511 & Paspalum $s p$ & Plicatula & Coxim, MS \\
\hline BRA-014851 & P. guenoarum & Plicatula & João Pinheiro, MG \\
\hline BRA-012866 & Paspalum sp & Plicatula & Caarapó, MS \\
\hline BRA-011681 & Paspalum sp & Plicatula & Campo Belo do Sul, SC \\
\hline BRA-005541 & Paspalum $s p$ & Plicatula & São Joaquim, SC \\
\hline BRA-009695 & Paspalum sp & Plicatula & Bella Vista, Paragua \\
\hline BRA-009407 & Paspalum $s p$ & Plicatula & Porto Velho, RO \\
\hline BRA-008311A & P. chacoense & Caespitosa & Corumbá, MS \\
\hline BRA-008630 & Paspalum sp & Plicatula & Lages, SC \\
\hline BRA-008486 & Paspalum sp & Plicatula & Planaltina, DF \\
\hline BRA-010464 & Paspalum sp & Plicatula & Aquidauana, MS \\
\hline BRA-009628 & Paspalum sp & Plicatula & Anastácio, MS \\
\hline BRA-009661 & Paspalum sp & Plicatula & Aquidauana, MS \\
\hline BRA-012483 & P.plicatulum & Plicatula & Pereira Barreto, SP \\
\hline BRA-009032 & P.plicatulum & Plicatula & Itaqui, RS \\
\hline BRA-012424 & Paspalum sp & Plicatula & Acreúna, GO \\
\hline BRA-009644 & Paspalum sp & Plicatula & Miranda, MS \\
\hline BRA-009687 & Paspalum sp & Plicatula & Bella Vista, Paragu \\
\hline BRA-012874 & Paspalum $s p$ & Plicatula & Caarapó, MS \\
\hline BRA-010537 & Paspalum sp & Plicatula & Cáceres, MT \\
\hline BRA-009652 & Paspalum sp & Plicatula & Miranda, MS \\
\hline
\end{tabular}


Continuação da Tabela 7...

\begin{tabular}{lccc}
\hline Acesso & $\begin{array}{c}\text { Espécie } \\
\text { Accession }\end{array}$ & $\begin{array}{c}\text { Grupoboties } \\
\text { Botanical group }\end{array}$ & $\begin{array}{c}\text { Local da coleta } \\
\text { Collection site }\end{array}$ \\
\hline BRA-008613 & Paspalum $s p$ & Plicatula & Curitibanos, SC \\
BRA-006572 & P. guenoarum & Plicatula & São Borja, RS \\
BRA-009113 & Paspalum $s p$ & Plicatula & Porto Alegre, RS \\
BRA-010260 & P. conspersum & Virgata & Miranda, MS \\
BRA-014770 & Paspalum $s p$ & Plicatula & Unaí, MG \\
BRA-014630 & Paspalum $s p$ & Plicatula & Curitibanos, SC \\
BRA-012416 & Paspalum $s p$ & Plicatula & Acreúna, GO \\
BRA-008923 & Paspalum $s p$ & Plicatula & São Gabriel, RS \\
BRA-012556 & Paspalum $s p$ & Plicatula & Campo Grande, MS \\
BRA-008508B & P. conspersum & Virgata & Vacaria, RS \\
BRA-012742 & Paspalum $s p$ & Plicatula & Caarapó, MS \\
BRA-003841 & P. chacoense & Caespitosa & Porto Murtinho, MS \\
BRA-009415 & Paspalum $s p$ & Plicatula & Cáceres, MT \\
BRA-010596 & Paspalum $s p$ & Plicatula & N.S.Livramento, MT \\
BRA-009229 & P. plicatulum & Plicatula & Guaíba, RS \\
BRA-013871 & P. ovale & Ovalia & Ponta Grossa, PR \\
BRA-003638 & Paspalum $s p$ & Plicatula & Goiatuba, GO \\
BRA-007498 & P. fasciculatum & Fasciculata & Cáceres, MT \\
BRA-008851 & Paspalum $s p$ & Plicatula & Arroio dos Ratos, RS \\
BRA-013951 & Paspalum $s p$ & Plicatula & Ponta Grossa, PR \\
BRA-015067 & P. maritimum & Caespitosa & Oiapoque, AP \\
BRA-001503 & P. oteroi & Plicatula & Corumbá, MS \\
BRA-011487 & P. nicorae & Plicatula & Bom Jesus, RS \\
\hline & & & \\
\hline & & &
\end{tabular}

produção de matéria seca total no ano com as obtidas no período seco e chuvoso. A correlação para a característica produção de matéria seca entre os períodos seco e chuvoso, também, mostrou linearidade significativa $(\mathrm{P}<0,01)$ nos dois primeiros e não-significativa no terceiro experimento. É conceitual ser maior a produtividade de matéria seca durante o período chuvoso em relação ao período seco, principalmente em gramíneas C4, como demostra SINGH et al. (1996) em Lasiurus scindicus, que apresentou eficiência de 9,3 a 20,8 kg MS/ha por milímetro de chuva, associada à eficiência térmica de 0,60 a $0,77 \mathrm{~kg} \mathrm{MS} / \mathrm{ha}$ por grau Celsius. A redução da sazonalidade também pode ser minimizada com uso de manejo varietal adequado ao sistema em uso, como sugerido por GARCIA-LOPEZ et al. (1995), e introdução de Chloris gayana cv. Callide, em pastagem já formada por Digitaria decumbens, visando a redução da sazonalidade da produção. Nesse caso, os autores obtiveram produtividade anual distribuída em $46 \%$ no período seco e o restante no período chuvoso em relação aos $30 \%$ de matéria seca produzida no período seco em pastagens somente com Digitaria decumbens.

Os resultados obtidos neste trabalho indicam que, em média, os acessos avaliados que apresentaram maiores produções no período chuvoso também apre- sentaram produções elevadas no ano (Tabela 6). Contudo, correlações positivas e significativas, embora em valores menores que as obtidas entre MSANO e MSCHUVA, foram obtidas entre MSCHUVA e MSSECO nos experimentos 1 e 2, indicando que ganhos de seleção para estabilidade na produtividade de matéria seca podem ser obtidos mais facilmente, quando a seleção for realizada com objetivo de elevar a correlação entre MSCHUVA com MSSECO do que quando objetiva reduzir a correlação entre MSANO com MSCHUVA.

Os acessos BRA-008630, BRA-009407, BRA003077 e BRA-006602, avaliados no presente trabalho, obtiveram maiores produções de matéria seca dentre os que apresentaram estabilidade produtiva para os períodos de seca e chuva, porém suas produtividades foram, em média, $15 \mathrm{t}$ MS/ha, bastante inferior às testemunhas utilizadas. A obtenção de cultivares com estabilidade em ambientes distintos $\mathrm{e}$ com elevada produtividade é dificultada por barreiras fisiológicas, como sensibilidade a fotoperíodo e baixas temperaturas.

\section{Conclusões}

Há variabilidade significativa para produção de biomassa entre os acessos avaliados no primeiro ano 
de implantação, sendo esta dependente de efeitos ambientais de ano e época.

A produção anual de matéria seca, na média dos acessos avaliados, foi proveniente de $70 \%$ do período de máxima precipitação pluvial e de $30 \%$ do período de precipitação pluvial mínima.

O quadrante formado pelas latitudes de $19^{\circ}$ a $22^{\circ} \mathrm{S}$ e longitudes de $47^{\circ}$ a $63^{\circ} \mathrm{W}$ apresenta $47 \%$ dos acessos selecionados, constituindo-se em área prioritária para futuras coletas para introdução de germoplasma de Paspalum na região Sudeste do Brasil.

\section{Referências Bibliográficas}

BATISTA, L.A.R., GODOY, R. Variabilidade intraespecífica em Paspalum notatum Fluegge. In: CONGRESSO NACIONAL DE GENÉTICA, 43, 1997, Goiânia. Anais...Ribeirão Preto: SBG, 1997, p.323.

BURTON, G.W. 1945. Dallisgras seed sources. J. Amer. Soc. Agron., 37(2):458-68.

BURTON, G.W. 1962. Conventional breeding of dallisgrass, Paspalum dilatatum Poir. Crop Sci, 2(6):491-494.

BURTON, G.W. 1967. A search for the origin of Pensacola bahiagrass. Econ. Botanic, 21(2):379-382.

CHANK, S.C. 1974. Ocorrência do vírus do enfezamento (P.S.V.) no capim pangola. R. Soc. Bras. Zootec., 3(2):225-31.

CHASE, A. 1937. New species of Paspalum from tropical America. J. Washington Acad. Sci., 27(4):143-146.

COSENZA, G.W. 1982. Resistência de gramíneas forrageiras a cigarrinha-das-pastagens Deois flavopictta (Stal 1854), 2ed. Planaltina, EMBRAPA-CPAC. 22p (EMBRAPACPAC, Boletim de Pesquisa, 7).

DIAS FILHO, M.B. 1982. Adaptação de gramíneas forrageiras em área originalmente de florestas da Amazônia Oriental Brasileira. Belém, EMBRAPA-CPATU. 3p. (EMBRAPACPATU, Pesquisa em andamento, 86).

GARCIA LOPEZ, R., FUNES, F., FERNANDEZ, J. 1995 Introduction of Rhodes Callide (Chloris gayana cv Callide) on established pastures and its effect on pasture stability and milk production. Cuba. J. Agric. Sci., 29(3):297-300.
GOMES, F.P. 1978. Curso de estatística experimental. Livraria Nobel, 8.ed. São Paulo: Ed. Nobel. p.405-426.

KALMBACHER, R.S., MULLAHEY, J.J., MARTIN, F.G et al. 1997. Effect of clipping on yield and nutritive value of "Suerte" Paspalum atratum. Agron. J., 89(3):476-81.

MANNETJE, L.T. 1961. A key based on vegetative characters of some induced species of Paspalum, S.1. CSIRO. (CSIRO. Technical Paper. 1).

PRIMO, A.T. 1975. CONDEPE constata casos de fotossensibilização em pasto de braquiária. Agricultura e pecuária, 605:3.

RODRIGUES, L.R.A. Estabelecimento de pastagens de Cynodon. In: SIMPÓSIO SOBRE MANEJO DA PASTAGEM, 15, 1998, Piracicaba. Anais... Piracicaba: FEALQ, 1998, p.115-50.

SCHULTZE-KRAFT, R. Recolecíon de plantas nativas con potencial forrajero. In: SIMPÓSIO SOBRE PLANTAS FORRAGEIRAS, 1979, Campo Grande (MS). Anais... Brasília: EMBRAPA/CENARGEN/BID, 1980, p.61-72 (EMBRAPA-CENARGEN. Documentos, 1).

SINGH, K.C., RAO, A.S., SINGH, H.P. 1996. Estimation of production potential and yield of sewan (Lasiurus sindicus) grass in Thar desert of Rajasthan. Ind. J. Agric. Sci., 66(7):413-417.

STRAPASON, E. Seleção de descritores na caracterização de germoplasma de Paspalum através de componentes principais. Piracicaba, SP. USP, 1997.95p. Dissertação (Mestrado em Estatística e Experimentação Agronômica) - Universidade de São Paulo, 1997.

TAKAYAMA, S.Y., FREITAS, P.M., PAGLIARINI, M.S. et al. 1998. Chromosome number in germplasm accessions of Paspalum (plicatula group) from different regions in Brazil. Euphytica, 99(2):89-94.

TISCHLER, C.R., BURSON, B.L. 1995. Evaluation different bahiagrass cytotypes for heat tolerance and leaf epicuticular wax content. Euphytica, 84(3):229-235.

Recebido em: 13/08/99

Aceito em: 12/08/99 